\title{
Transbronchial Pulmonary Biopsies
}

\author{
G. Casoni, C. Gurioli, V. Poletti
}

Monaldi Arch Chest Dis 2011; 75: 1, 39-41.

Keywords: Transbronchial lung biopsy, Diffuse parenchimal lung disease, High definition computerised tomography, Peripheral nodular lung lesion, Endoscopic ultrasound.

Dipartimento di Malattie del Torace, Ospedale Morgagni-Pierantoni, Forlì, Italy.

Correspondence: Dr. Gian Luca Casoni, Dipartimento di Malattie del Torace, Ospedale Morgagni-Pierantoni, Via Forlanini 34, 47100 Forli, Italy; e-mail: casonig1970@libero.it

The ability to obtain the pulmonary tissue without the need for the patient to undergo a surgical pulmonary biopsy is an important step forward in the diagnosis of the lung diseases.

The bronchoscopic pulmonary biopsy, defined also as transbronchial lung biopsy (TBLB), is a procedure with low morbidity and mortality. In fact the main indications for performing TBLB are the evaluation of peripheral nodular lung lesions or diagnosis of diffuse parenchymal lung disease.

The first description of TBLB is attributed to Andersen et al. [1], as a result of their observation that the specimens of bronchial biopsies of the small airways collected during a rigid bronchoscopy often contained pulmonary parenchyma. The forceps for TBLB collect the pulmonary tissue through the bronchi and enable specimens from the centrolobular regions to be obtained [2]. The disorders that are centred around the terminal and respiratory bronchioles (respiratory bronchiolitis, tuberculosis, lobular infectious pneumonia, cellular bronchiolitis) or significantly involve these structures (organising pneumonia) or those that are distributed along the lymphatic routes (sarcoidosis, carcinomatous lymphangitis) may be easily sampled by forceps. Multiple biopsy samples are required for a good diagnostic yield [3]. Most studies [3-7] have shown that there is a direct correlation between the number of samples obtained per TBLB and the overall diagnostic yield (i.e $38 \%$ with one to three tissue fragments vs. $69 \%$ with six to ten, $p<0.01)$. Therefore if the procedure proceeds normally, it is reasonable to perform 7-8 biopsies (fragments of valid tissue!) for examination. A greater number of biopsies may be necessary if a part of tissue is likely to be sent away for special stains or studies. Large forceps produce bigger specimens. Loube et al. [6] prospectively compared the diagnostic yield of transbronchial biopsies using large and small forceps (cup sizes, $3 \times 2$ x 0.9 vs. 2 x 1.5 x $0.6 \mathrm{~mm}$ respectively). Large forceps obtained significantly more tissue than the small forceps [ 16 of 22 patients $(73 \%)$ vs. 6 of 22 patients $(27 \%), p<0.05$, with no alveolar tissue obtained in 5 patients]. Further, if larger forceps are not withdrawn through the operative channel, crash artefacts may also be avoid [8] improving diagnos- tic yield. The main complication of TBLB is bleeding; less frequent complications are pneumothorax, hypoxemia, or cardiac arrhythmias during the procedure. Bleeding occurs to some degree in virtually all TBLB procedures and in some cases can be substantial. The risk of bleeding is the main limiting factor in obtaining more or larger biopsy samples. The suction channel of the typical fiber-bronchoscope is only $2 \mathrm{~mm}$ in diameter, and the volume of blood that can be suctioned through the channel is hence limited. Therefore, a bronchoscopist should be also trained in the use of a rigid bronchoscope to control any bleeding post TBLB.

The effective execution of the TBLB begins with an adapted selection and preparation of the patient which includes the aims and risks of the procedure. The bronchoscopy and the TBLB should be performed in an appropriate and well equipped room for the effective monitoring of the arterial pressure and oxygen saturation. Moreover the equipment for the additional oxygen therapy, the cardiopulmonary resuscitation must be available. The use of radioscopy during the TBLB is debated; however the TBLB is still usually performed with fluoroscopic guidance as the rate of pneumothorax is reduced when fluoroscopy is used [9]. The bronchoscopist should be trained in the employment of radioscopic equipment or have a staff trained during the procedure. The TBLB can be performed both by fiber-bronchoscope or rigid bronchoscope in general anesthesia.

\section{Indications}

The main indications for performing TBLB are the evaluation of peripheral nodular lung lesions and the diagnosis of diffuse parenchymal lung disease.

\section{Peripheral localised lesions of the lung}

Peripheral nodular pulmonary lesions can be diagnosed by TBLB. It has been demonstrated that the dimension of the pulmonary nodule (in particular if this is $<$ to $2 \mathrm{~cm}$ ) represents the limiting factor of the diagnostic yield of TBLB [10]. However it has been shown an improvement of diagnostic yield of the TBLB in diagnosis of a peripheral pul- 
monary nodule with diameter $<3 \mathrm{~cm}$ using Endobronchial Ultrasound-Driven Biopsy (EBUS) [11]. In particular, EBUS-guided TBLB is a safe and very effective method for solitary pulmonary nodules that cannot be visualized by fluoroscopy. The procedure may increase the yield of endoscopic biopsy in patients with these nodules and avert the need for surgical procedures [12]. Then, the bronchus sign at a fourth-order bronchus is valuable in predicting the success of transbronchial biopsy. The presence of the sign on CT may be useful in determining if the work-up should include transbronchial biopsy or transthoracic needle aspiration in patients with peripheral lung lesions [13].

\section{Diffuse parenchymal lung disease}

TBLB may be considered as the first choice for diagnosis of diffuse parenchymal lung disease. Histological examinations should be performed routinely. The decision as to the type, size, and site of the biopsy sample is determined by the level of diagnostic certainty before performing lung biopsy, the suspected nature, distribution and extent of the disease, and the patient's performance status. The introduction of the high definition computerised tomography (HRCT) has enormously improved the resolution of pulmonary spaces and it has allowed the lesions to be better defined the lesions through the interpretation of morphologic characteristics and anatomical distribution (analysis for patterns) particularly in the context of diffuse parenchymal lung disease [14]. HRCT is very useful in predicting the yield of TBLB based on the anatomic distribution and appearance of any abnormalities in particular with the presence of some HRCT patterns such as nodular, alveolar consolidations, ground glass, tree in bud and thickening of the interlobular septa (peri-lymphatic pattern) in which the diagnostic yield of the TBLB is better than others patterns (i.e. reticular pattern) [15]. The first step in the diagnosis of diffuse parenchymal lung disease is to divide and differentiate between immuno-compromised and immuno-competent patients [16, 17].

Immunocompromised patients who develop diffuse lung disease are usually acutely ill with fever, cough, shortness of breath, and often with progressive hypoxemia $[16,17]$. A prompt diagnosis seems, therefore, to be crucial for a better survival. In cases in which a TBLB is deemed necessary and is feasible (absence of significant coagulation disorder, platelet $>50,000 /$ microliter) Bronchoscopic procedures should be performed under general anaesthesia through a rigid bronchoscope or an orotracheal or 'nasotracheal' tube in order to better control the ventilation and bleeding [8]. Combined BAL and TBLB may also be performed safely in mechanically ventilated patients [22]. Non-specific pathological changes are common in TBLB specimens in these patients but if they are interpreted in the context of a specific clinical setting and of HRCT scan patterns they may contribute to the definition of a specific diagnosis (in particular drug-related lung toxicity, alveolar haemorrhage and vasculitis, alveolar pro- teinosis) $[18,19]$. Also infections may have nonspecific changes as the sole morphological features. Special stains are, therefore, mandatory when TBLB specimens are evaluated in immunocompromised patients. Special stains are also useful for the classification of epithelial tumours and necessary to better identify lymphoid infiltrates. Remarkable interest must be placed on the impact of invasive diagnostic techniques (TBLB) on the survival in the immunocompromised patients [20]. A reduction of mortality in those patients that were diagnosed at an earlier stage has been demonstrated [21]. On other hand a delay of $>5$ days in establishing a specific diagnosis, was directly correlated with an increased mortality.

In the immunocompetent patients TBLB may be considered as the first choice. Monomorphous histological patterns (organising pneumonia, eosinophilic pneumonia, diffuse alveolar damage with or without eosinophils, alveolar haemorrhage with or without capillaritis, alveolar proteinosis) [18, 19] are easily identifiable in small lung fragments but they are more or less non-specific and are considered diagnostic only in agreement with clinical, radiological and other laboratory data. Specimens obtained through TBLB should be serially sectioned and immunohistochemical investigations should be encouraged for example, Langerhans cell histiocytosis, lymphangioleiomyomatosis, sarcoidosis, carcinomatous or malignant soft tissue metastases, and lymphoproliferative disorders are better defined using specific monoclonal antibodies (CD1a, Langerhin, HMB-45, CD68, TTF-1 or CDX-2 immunohistochemical expression). The tiny specimens obtained by TBLB may not be sufficient in cases in which the morphological features are characteristic only in large volumes of tissue (usual interstitial pneumonitis, desquamative interstitial pneumonitis, nonspecific interstitial pneumonitis, fibrosing type). Usually, TBLB specimens are usually considered too small and non-representative to allow the diagnosis of the usual interstitial pneumonia (UIP) pattern, although recently [22] the presence of patchwork fibrosis along with honeycomb changes and/or the presence of fibroblastic foci were shown to be present in TBLB specimens in $32 \%$ of patients with histologically proved UIP. Furthermore, we have also shown [8] that the characteristic histological features of diffuse parenchymal lung disease/IIP may be identified on TBLB specimens more often than previously appreciated using more large flexible forceps (as modified gastroenterological forceps called "Jumbo" forcep) via rigid bronchoscopy. Generous TBLB may provide good specimens for a morphological diagnosis of pattern called nonspecific interstitial pneumonia (NSIP), cellular variant. This pathological diagnosis is acceptable in specific clinical settings (polymyositis-dermatomyositis-related interstitial lung disease, drug toxicity) but it might also be of clinical value in cases of unknown aetiology.

\section{Contraindications}

Evaluation of the patient before the procedure is crucial. TBLB is contraindicated in the presence of bleeding abnormalities. An international nor- 
malised ratio (INR) greater than 1.5 is an absolute contra-indication; successful TBLB can be carried out if the INR is less than 1.5. If a patient is taking warfarin, it can usually be withheld for 4 to 5 days to allow the INR to slowly decrease to a safer range. TBLB is also contraindicated if the platelet count is less than $50000 / \mu \mathrm{L}$ but the platelet count can be increased relatively quickly with platelet transfusions prior to the procedure. Also, platelet dysfunction is a relative contra-indication to biopsy. There is insufficient data on antiplatelet agents such as clopidogrel, but most bronchoscopists are hesitant to perform a TBLB if the patient is using this agent. Other contra-indications for bronchoscopy and TBLB are severe hypoxemia, uncontrolled cardiac arrhythmias, unstable angina, and severe asthma or exacerbation of chronic obstructive pulmonary disease.

\section{Recommendations}

\section{- The main indications for performing} TBLB are the evaluation of peripheral nodular lung lesions or diagnosis of diffuse parenchymal lung disease (Grade B).

- TBLB may be considered as the first choice for diagnosis of diffuse parenchymal lung disease. Histological examination should be routinely performed. HRCT is very useful in predicting the yield of TBLB based on the anatomic distribution and appearance of any abnormalities (Grade B).

- The HRCT has demonstrated useful to increase the diagnostic accuracy of TBLB in presence of some HRCT patterns as nodular, alveolar consolidations, ground glass, tree in bud, thickening of the interlobular septa (peri-lymphatic pattern) and presence of broncus sign (Grade B).

- In order to obtain a satisfacory diagnostic yield it is reasonable to carry out 7-8 biopsies (fragments of valid tissue!) for examination (Grade B).

- The size of lung nodules (especially if it is $<2 \mathbf{~ c m}$ ) is the limiting factor in the diagnostic yield of TBLB. The use of endoscopic ultrasound (EBUS) significantly improves the diagnostic yield of TBLB in the presence of a peripheral pulmonary nodule with a diameter less than $3 \mathrm{~cm}$ (Grade B).

\section{References}

1. Andersen HA, Fontana RS, Harrison EG. Transbronchoscopic lung biopsy in diffuse pulmonary disease. Dis Chest 1965; 48: 187-192.

2. Poletti V, Patelli M, Ferracini R, Simonetti M, Spiga L. Transbronchial lung biopsy in infiltrative lung disease.
The importance of the pathologic approach. Sarcoidosis 1988; 5: 43-50.

3. Descombes E, Gardiol D, Leuenberger P. Transbronchial lung biopsy: An analysis of 530 cases with reference to the number of samples. Monaldi Arch Chest Dis 1997; 52: 324-329.

4. Hopkins PM, Aboyoun CL, Chhajed PN, et al. Prospective analysis of 1,235 transbronchial lung biopsies in lung transplant recipients. J Heart Lung Transplant 2002; 21: 1062-7.

5. Curley FJ, Johal JS, Burke ME, Fraire AE. Transbronchial lung biopsy: Can specimen quality be predicted at the time of biopsy? Chest 1998; 113: 1037-1041.

6. Loube DI, Johnson JE, Wiener D, Anders GT, Blanton HM, Hayes JA: The effect of forceps size on the adequacy of specimens obtained by transbronchial biopsy. Am Rev Respir Dis 1993; 148: 1411-1413.

7. Becker HD, Shirakawa T, Tanaka F, Muller KM, Herth F. Transbronchial (transbronchoscopic) lung biopsy in immunocompromised patient; in Strausz J (ed): Pulmonary Endoscopy and Biopsy Techniques. Eur Respir Monogr 1998, vol 3, pp 193-208.

8. Casoni GL, Gurioli C, Chhajed PN, et al. The value of transbronchial lung biopsy using jumbo forceps via rigid bronchoscope in diffuse lung disease. Monaldi Arch Chest Dis 2008; 69: 59-64.

9. Prakash UB, Offord KP, Stubbs SE. Bronchoscopy in North America: the ACCP survey. Chest 1991; 100: 1668-75.

10. Baaklini WA, Reinoso MA, Gorin AB, Sharafkaneh A, Manian P. Diagnostic yield of fiberoptic bronchoscopy in evaluating solitary pulmonary nodules. Chest 2000; 117: 1049-1054.

11. Paone G, Nicastri E, Lucantoni G, et al. Endobronchial Ultrasound-Driven Biopsy in the diagnosis of peripheral lung lesions. Chest 2005; 128: 3551-3557.

12. Herth FJ, Eberhardt, Becker HD and Ernst A. Endobronchial ultrasound-guided transbronchial lung biopsy in fluoroscopically invisible solitary pulmonary nodules. Chest 2006; 129; 147-150.

13. Gaeta M, Pandolfo I, Volta S, et al. Bronchus sign on CT in peripheral carcinoma of the lung: value in predicting results of transbronchial biopsy. AJR 1991; 157: 1181-1185.

14. Zompatori M, Sverzellati N, Poletti V, et al. High-resolution CT in diagnosis of diffuse infiltrative lung disease. Semin Ultrasound CT MR 2005; 26: 332-347.

15. Colby TV, Swensen SJ. Anatomic distribution and histopathologic patterns in diffuse lung disease: correlation with HRCT. J Thorac Imaging 1996; 1: 1-26.

16. Poletti V, Costabel U, Semenzato G. Pulmonary complications in patients with haematological disorders: pathobiological bases and practical approach. Semin Respir Crit Care Med 2005; 26: 439-444.

17. Poletti V, Trisolini R, Tura S. Pulmonary disease in patients with hematologic malignancies. Sarcoidosis Vasc Diffuse Lung Dis 2002; 19: 29-45.

18. Poletti V, Cazzato S, Minicuci N, et al. The diagnostic value of bronchoalveolar lavage and transbronchial lung biopsy in cryptogenic organizing pneumonia. Eur Respir J 1996; 9: 2513-2516.

19. Lacasse Y, Fraser RS, Fournier M, Cormier Y. Diagnostic accuracy of transbronchial biopsy in acute farmer's lung disease. Chest 1997; 112: 1459-1465.

20. Mayaud C, Cadranel J. A persistent challenge: the diagnosis of respiratory disease in the non-AIDS immunocompromised host. Thorax 2000; 55: 511-517.

21. Rano A, Agusti C, Benito N, et al. Prognostic factors of non-HIV immunocompromised patients with pulmonary infiltrates. Chest 2002; 122: 253-261.

22. Berbesku EA,Katzenstein AL, Snow JL, et al. Transbronchial biopsy in usual interstitial pneumonia. Chest 2006; 129: 1126-1131. 